\title{
INTERESANTE NUEVA ESPECIE DE GEOCHARIS EHLERS, 1883 DE ESPAÑA (COLEOPTERA, CARABOIDEA, TRECHIDAE, ANILLINI) *
}

\author{
J. P. Zaballos $(* *)$
}

\begin{abstract}
RESUMEN
Se describe una nueva especie de Anillini de Cáceres (España): Geocharis leoni sp. n. Se compara morfológicamente con las especies vecinas y se describe y comenta de forma especial la morfología del edeago: el parámero izquierdo es largo, ancho y plano, y su parte distal es muy especial, ya que las setas terminales se han transformado en tres escamas laminares y membranosas. Además, existen otras tres lamelas membranosas entre el parámero izquierdo y el lóbulo medio. En la superficie dorsal del lóbulo medio hay varias líneas microserruladas y sensilas de tipo campaniforme.
\end{abstract}

Palabras clave: Coleoptera, Caraboidea, Anillini, Geocharis leoni sp. n., España.

\author{
ABSTRACT \\ A interesting new Geocharis Ehlers, 1883 from Spain \\ (Coleoptera, Caraboidea, Trechidae, Anillini)
}

A new species of Anillini from Cáceres (Spain): Geocharis leoni sp. n. is described. It is compared with related species and the morphology of the aedeagus is specially studied: the left paramere is long, large and flattened, and the apex is very unusual, because the terminal setae are transformated in three lamellar and membranous scales. In addition, other three long membranous lamella are present between the left paramere and the median lobe. The dorsal surface of the median lobe has some microserrate lines and campaniform sensilas.

Key words: Coleoptera, Caraboidea, Anillini, Geocharis leoni sp. n., Spain.

\section{Introducción}

En la primavera de 1990, dentro de una campaña de prospecciones edáficas en Extremadura, Luis G. Gómez obtuvo un ejemplar del género Geocharis Ehlers, 1883 en un talud de un arroyo, al norte de la provincia de Cáceres, en la sierra de Santa Bárbara. Una segunda visita a la localidad, siete años más tarde, permitió capturar ocho ejemplares más. Estas nuevas capturas han posibilitado realizar un detallado estudio morfológico y comprobar que se trataba, tal como habíamos supuesto, de una nueva especie que se describe a continuación. El estudio del órgano copulador masculino ha resultado ser especialmente sugerente, ya que presenta ciertas estructuras no descritas hasta la fecha dentro de los carábidos.

* Este trabajo ha sido subvencionado por los proyectos de la DGICYT PB 92-0121 (Fauna Ibérica III) y DGES PB05-0235 (Fauna Ibérica IV).

** Dpto. Biología Animal I. Facultad de Biología. Universidad Complutense de Madrid. 28040 Madrid. 

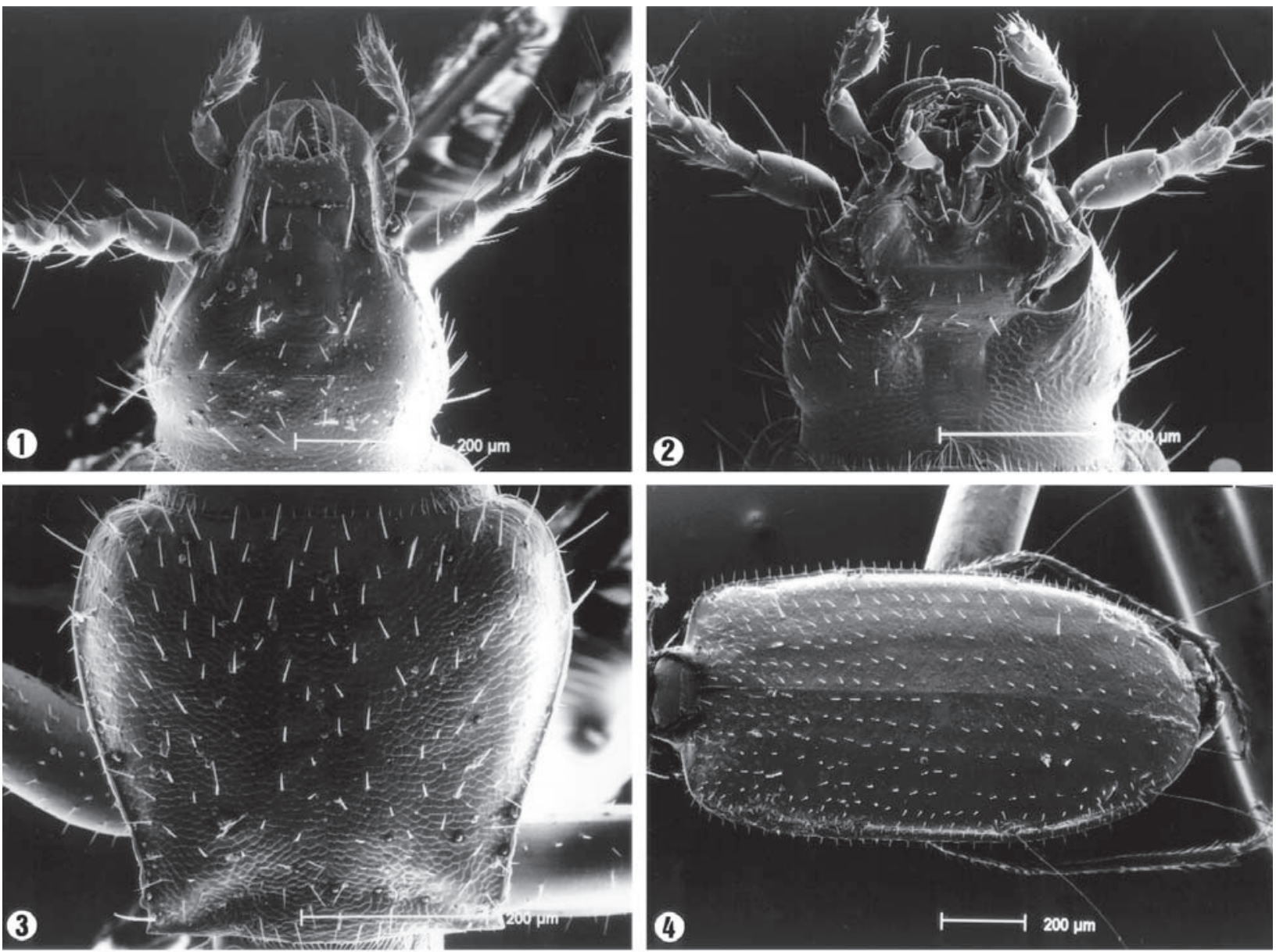

Figs. 1-4.-Geocharis leoni sp. n.: 1) cabeza, 2) piezas labiales, 3) pronoto, 4) élitros.

Figs. 1-4.-Geocharis leoni sp. n.: 1) head, 2) mouth pieces, 3) pronotum, 4) elytra.

\section{Material y métodos}

El material fue obtenido a partir de tierra tomada de taludes, transportada al laboratorio en sacos de plástico y colocada en embudos de Berlese con líquido Scheerpeltz en los recipientes de recogida.

El estudio morfológico fue realizado utilizando microscopía óptica tradicional y fotografías obtenidas con un microscopio electrónico de barrido, modelo Philips XL-20, con ejemplares metalizados con bio-rad SC-515 por el método "Sputter coating" y velocidades de aceleración de 15 kilovoltios.

El montaje de los ejemplares en cartulinas y en preparaciones microscópicas fue realizado con resina D.M.H.F. (Bameul, 1990). Estas preparaciones están montadas en cartulinas con ventanas de cristal junto con cada ejemplar.
Los dibujos de las genitalias se realizaron con cámara clara en un microscopio Zeiss 474620-9900.

\section{Descripción taxonómica}

Geocharis leoni sp. n.

SERIE TíPICA: Holotipo: $10^{7}$, NW Santa Cruz de Paniagua, 475 m, Cáceres, U.T.M.: 29TQE2455, 14-III-1997, J.P.Zaballos leg. (en la Coll. J. P. Zaballos). Paratipos: $30^{x} \sigma^{x}$ y 4 ㅇ 우, mismos datos que el holotipo (un ejemplar de cada sexo metalizados). $1 \sigma^{\pi}$, ídem, 7-V-1990, Luis G. Gómez leg. (en las colecciones del Museo Nacional de Ciencias Naturales de Madrid ${ }^{\circ}$ cat. 12471 y en la Coll. J. P. Zaballos).

Diagnosis: Coleóptero Trechidae ciego, longitud 1,9-2,2 mm, deprimido y despigmentado, de 

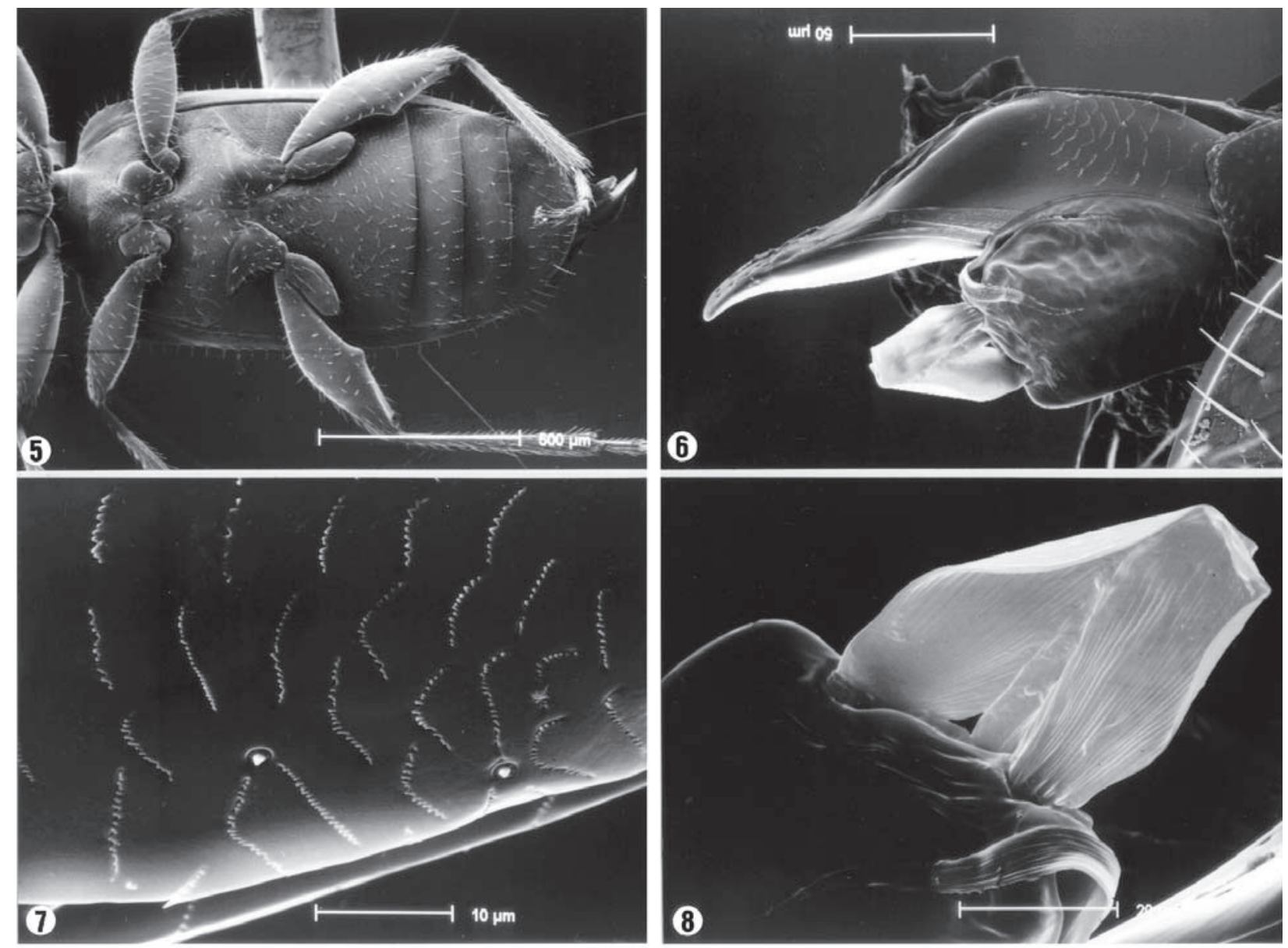

Figs. 5-8.-Geocharis leoni sp. n.: 5) macho en vista ventral, 6) Lóbulo medio del edeago y parámero izquierdo, 7) detalle de la superficie dorsal del lóbulo medio, 8) ápice del parámero izquierdo.

Figs. 5-8.- Geocharis leoni sp. n.: 5) male in ventral view, 6) median lobe of aedeagus and left paramere, 7) detail of dorsal surface of median lobe, 8) apex of the left paramere.

aspecto grácil y color castaño claro, con el tegumento microrreticulado, cubierto de una pubescencia fina y dispersa por toda la superficie corporal salvo en los élitros, donde está alineada. Élitros con dos pares de setas discales: las anteriores y las posteriores, sin estrías. Machos con el primer artejo de los tarsos anteriores ligeramente dilatado y fémures de las patas posteriores con un diente en su arista interna. Edeago con el parámero izquierdo largo, ancho y plano, acabado en tres escamas laminares y membranosas además de otras tres lamelas membranosas entre el parámero izquierdo y el lóbulo medio. El parámero derecho normal, bisetulado.
DESCRIPCIÓN: Cabeza (fig. 1) más larga $\left(0^{7}\right.$ : $0,51 \mathrm{~mm}$, o : $0,41 \mathrm{~mm})$ que ancha $\left(\sigma^{7}: 0,43 \mathrm{~mm}\right.$, o : $0,36 \mathrm{~mm})$, rebordeada hasta la segunda seta supraocular, cuello estrecho bien marcado. Antenas y piezas labiales (fig. 2) sin particularidades dentro del género. Quetotaxia cefálica formada por 8 setas labrales, 2 clipeales, 2 frontales, 2 supraoculares, 46 laterales (en la parte más ancha de la cabeza, donde termina el reborde lateral) y 2 temporales.

Pronoto (fig. 3) 1,2 veces más ancho $\left(\sigma^{7}: 0,54\right.$ $\mathrm{mm}$, o : $0,44 \mathrm{~mm})$ que largo $\left(0^{\top}: 0,46 \mathrm{~mm}\right.$, ㅇ : 0,36 $\mathrm{mm}$ ), con la parte basal estrechada, apenas sinuada hacia los ángulos posteriores, que son casi rectos y están precedidos de un par de dientes apenas visi- 

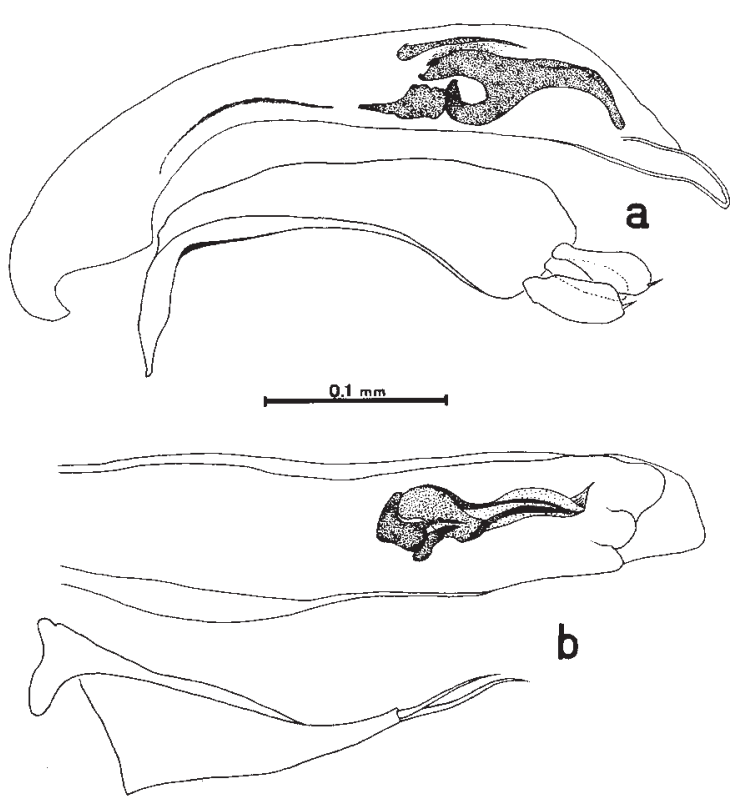

Fig. 9.- Geocharis leoni sp. n.: lóbulo mediano del edeago y parámero izquierdo en vista lateral (a) y lóbulo mediano del edeago y parámero derecho en vista dorsal (b).

Fig. 9.- Geocharis leoni sp. n.: median lobe of aedeagus and left paramere in lateral view (a) and median lobe of aedeagus and right paramere in dorsal view (b).

bles. Plano en el disco y deprimido en las fosetas basales. Reborde lateral con 2 setas marginales anteriores y dos posteriores o basales insertadas casi en los ángulos. En el resto del pronoto se aprecia una fina pubescencia repartida por el disco, en la proximidad de los ángulos anteriores hay 2-3 setas más largas.

Élitros (fig. 4) casi dos veces más largos $\left(\sigma^{7}\right.$ : $1,23 \mathrm{~mm}$, o : $1,13 \mathrm{~mm})$ que anchos $\left(\sigma^{7}: 0,62 \mathrm{~mm}\right.$, ㅇ : $0,59 \mathrm{~mm}$ ), subparalelos con el margen posterior ovalado. Superficie dorsal deprimida, con un punteado fino, apenas visible, en el tercio anterior, entre la microescultura. Aunque carece de estrías la pubescencia está claramente alineada en el centro de los élitros. Hombros redondeados, sin formar ángulo aparente. Margen lateral estrecho en toda su longitud, orlado de cortas setas; en él se insertan las setas de la serie umbilicada marginal: las tres primeras en la zona humeral, la primera separada de la $2^{\mathrm{a}}$ y $3^{\mathrm{a}}$, la $4^{\mathrm{a}}$ aislada, a la mitad de distancia de la $3^{\mathrm{a}}$ que de la $5^{\mathrm{a}}$, que con la $6^{\mathrm{a}}$ forma un par separado del grupo posterior, el cual está formado por la $7^{\mathrm{a}}, 8^{\mathrm{a}}$ y $9^{a}$. Las grandes setas de la serie umbilicada son la $2^{\mathrm{a}}, 6^{\mathrm{a}}$ y $9^{\mathrm{a}}$, las setas $3^{\mathrm{a}}, 5^{\mathrm{a}}, 7^{\mathrm{a}}$ y $8^{\mathrm{a}}$ están ligeramente desplazadas hacia el interior. Además, en los élitros, existen un par de setas basales con poro, dos pares de setas discales, las anteriores y las posteriores, y un par de setas apicales.

Patas con dimorfismo sexual: los machos con el primer artejo de los tarsos anteriores ligeramente dilatado y algunas faneras adhesivas, y fémures de las patas posteriores (fig. 5) con un neto diente en el centro de la arista interna.

Órgano copulador masculino (figs. 6, 7, 8, 9) con el lóbulo medio de $0,4 \mathrm{~mm}$ de longitud, fino, apenas arqueado, con la lámina apical ancha. Superficie dorsal del mismo con una serie de líneas curvas microserruladas (microtriquias) y sensilas campaniformes (figs. 6, 7). Endofalo con una pieza copuladora tal como que se aprecia en la figura 9. Parámero derecho normal, acabado en dos setas (fig. 9b), parámero izquierdo ancho y plano, con la parte distal en forma de paleta con tres escamas membranosas distales, sustituyendo a las setas normales (figs. 6, 8,9). Además, entre el lóbulo medio y el parámero izquierdo, existen tres lamelas membranosas, largas y finas (figs. 6, 8).

Armadura genital femenina (fig. 10) similar a la de otras especies del género (Zaballos \& Jeanne, 1987), cada gonocoxito con una gruesa seta espiniforme en la arista externa, visible ventralmente, una seta espiniforme en la parte dorsal, y una seta sensorial, fina y larga, en la arista interna, lejos del ápice. Conducto espermatecal corto que vierte a la espermateca, que es esférica $(0,036 \mathrm{~mm}$ de diámetro). Glándula espermatecal alargada y, salvo una zona membranosa en la parte central, escasamente esclerotizada. La glándula vierte en la espermateca mediante un corto y estrecho conducto.

Dimorfismo sexual: además del primer artejo dilatado en las patas anteriores y la existencia de fémures con un diente (fig. 5) en las patas posteriores de los machos, el tamaño de estos $(2,2 \mathrm{~mm})$ es superior al de las hembras $(1,9 \mathrm{~mm})$.

ETIMOlogía: la nueva especie está dedicada, con un afectuoso agradecimiento, a mi amigo el Doctor José Luis León Aguado.

HÁBITAT: La serie típica fue obtenida con aparatos de Berlese de la tierra cogida en un talud arenoso de las orillas de un arroyo de aguas temporales, en una región con características climáticas mediterráneas.

AfINIDADES: La nueva especie está muy bien definida por la estructura del órgano copulador 
masculino, en especial por la forma del parámero izquierdo. No obstante, por la presencia de dos pares de setas discales (anteriores y posteriores) y por la inexistencia de estrías elitrales marcadas, Geocharis leoni sp. n. debe relacionarse con $G$. amicorum Zaballos, 1998, G. iborensis Zaballos, 1990 y G. femoralis Coiffait, 1968, pero se diferencia bien de ellas por la presencia de un diente mediano en la arista interna de los fémures posteriores de los machos, forma general esbelta y talla superior a $1,8 \mathrm{~mm}$.

La existencia de dichos dientes en los fémures posteriores de los machos la relaciona con G. cordubensis Dieck, 1869, de la que se diferencia bien por la posición de las setas discales.

Desde el punto de vista corológico, las especies más próximas son G. julianae Zaballos, 1989, G. iborensis Zaballos, 1990 y G. ruiztapiadori Zaballos, 1996, de las que se diferencia fácilmente utilizando la clave ya publicada (Zaballos, 1998).

Sin duda, la más afín es la ya mencionada $G$. iborensis, con la que comparte talla, aspecto general, dimorfismo sexual (con matizaciones que ayudan precisamente a separarlas en las patas posteriores de los machos) y la genitalia, salvo el lo referido al parámero izquierdo del órgano copulador masculino (ver comentario final).

\section{Comentario final}

Al margen del interés que puede suponer la descripción de esta nueva especie, lo más interesante que cabe resaltar de Geocharis leoni sp. n. es la estructura del órgano copulador masculino.

Ya con microscopía óptica tradicional se pudo observar la peculiar forma del estilo izquierdo: largo, ancho y acabado en tres escamas membranosas. Pero el estudio del mismo, con microscopía electrónica de barrido, ha posibilitado una mayor aproximación desde el punto de vista morfológico.

Además de las sensilas campaniformes (figs. 6, 7) (presentes en la superficie dorsal y lateral externa del lóbulo mediano y concentradas en mayor número en el ápice), hay una serie de procesos cuticulares que forman líneas curvas microserradas (microtriquias), dirigidas hacia la parte posterior, lo que permite la penetración en la vagina y dificulta la extracción del pene.

Con respecto al parámero izquierdo (figs. 6,8), se pudo comprobar la existencia de las tres escamas membranosas distales y observar, además, la existencia de otras tres lamelas membranosas (invisibles al microscopio óptico), muy finas y largas, que

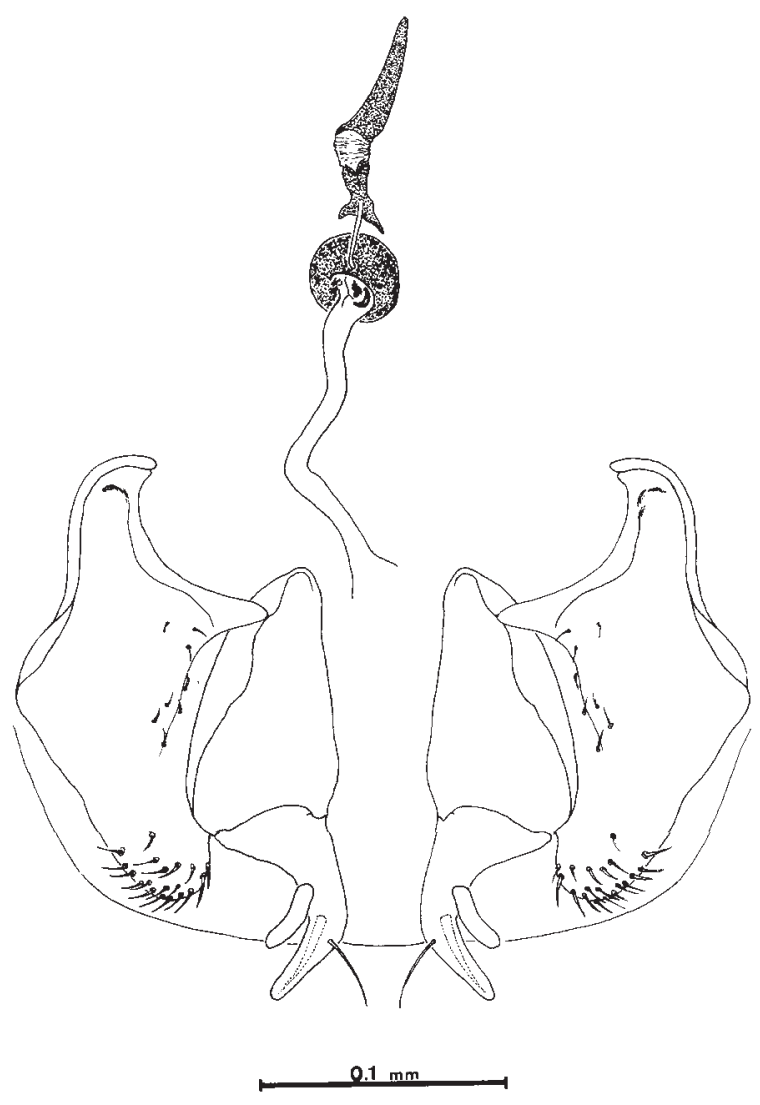

Fig. 10.- Geocharis leoni sp. n.: armadura genital femenina y complejo espermatecal en visión ventral.

Fig. 10.-Geocharis leoni sp. n.: ventral view of female genital shield and spermathecal complex.

se sitúan entre el parámero izquierdo y el lóbulo mediano. Una de ellas (que en las fotos aparece plegada sobre la parte distal del parámero) parece formar parte del borde superior del mismo, pero no se ha podido comprobar la inserción basal de las otras dos (adheridas sobre el lóbulo medio en las fotos).

Aunque dentro de los carábidos en general, y en los Stylifera en particular, es bastante frecuente la existencia de parámeros anchos, bien es cierto que este tipo de terminación sensorial es desconocida dentro del grupo. Según Jeannel (1955), es frecuente la existencia de divergencias morfológicas, en las setas de los parámeros, entre especies filéticamente próximas que habitan en los mismos biotopos, asegurando de esta forma un aislamiento reproductor precigótico de tipo sensorial.

La especie más próxima a la nueva especie, tanto corológica como morfológicamente, es $G$. 
iborensis, y tal como cabría esperar tiene el órgano copulador masculino (Zaballos, 1990) muy parecido a la nueva especie, salvo en lo que a los estilos se refiere.

Este tipo de aislamiento ya había sido mencionado anteriormente en un grupo de especies del género Typhlocharis (Zaballos \& Farinós, 1995), pero referido a una serie de dimorfismos sexuales que aseguran un aislamiento más de tipo mecánico que sensorial. En el caso de los Geocharis que nos ocupa ocurren ambas situaciones, ya que también presentan dimorfismo sexual en las patas posteriores de los machos: fémures dentados en G. leoni sp. n. y tibias serruladas en G. iborensis.

Estas llamativas estruturas morfológicas, bien de tipo sensorial (setas y escamas de los parámeros) o de tipo mecánico (patas posteriores de los machos con fémures o tibias modificadas, fosetas ventrales en las hembras, etc ), que sirven para evitar la cópula entre especies afines, proporcionan evidentes diferencias morfológicas, entre dichas especies, que deben interpretarse como elementos de clara relación filética entre ellas, y no todo lo contrario.

La combinación del desconocido grupo de los Anillini y el microscopio electrónico, con el apoyo de una sólida base morfológica, está abriendo una nueva dimensión y un nuevo concepto sistemático del grupo.

\section{AGRADECIMIENTOS}

A D. Luis G. Gómez, primer recolector de la especie, por la cesión del material para su estudio y a José Bedoya, del Museo Nacional de Ciencias Naturales de Madrid, por su asistencia en la realización de fotografías al microscopio electrónico de barrido.

\section{Referencias}

BAmeul, F., 1990. Le DMHF: un excellent milieu de montage en entomologie. L'Entomologiste, 46(5): 233-239

JEANNEL, R., 1955. L'édéage, initiation aux recherches sur la systématique des Coléoptères. Publications du Muséum National d'Histoire Naturelle, $\mathrm{n}^{\circ} 16$. Paris. $155 \mathrm{pp}$.

Zaballos, J.P., 1990. Un nuevo Geocharis de Extremadura (España) (Coleoptera, Trechidae). Boll. Mus. Reg. Sci. Nat. Torino, 8(2): 403-409.

Zaballos, J.P., 1998. Un nuevo Geocharis de Almería (Coleoptera, Caraboidea, Trechidae, Anillini). Zoologica Baetica, [1997], 8: 171-180.

Zaballos, J.P. \& Farinós, G. P., 1995. Systematics of the genus Typhlocharis Dieck: The T. gomezi species group (Coleoptera: Caraboidea: Trechidae). Coleopt. Bull., 49(1): 89-95.

Zaballos, J.P. \& JEAnNe, C., 1987. Etude systematique du Genre Geocharis (Col. Trechidae, Anillini) et description d'une nouvelle espèce. Bull. Soc. Linn. Bordeaux, 15(2): 81-92. 